\title{
Perfil sociodemográfico familiar e clínico de crianças com disfunções neurológicas atendidas no programa de intervenção precoce
}

\author{
Familial and clinical sociodemographic profile of children with \\ neurological dysfunction treated in the early intervention program \\ Perfil sociodemográfico familiar y clínico de niños con disfunciones \\ neurológicas atendidas en el programa de intervención precoz \\ Natália de Fátima Pereira MEIRELES ${ }^{1}$ \\ Paulo Henrique Meira DUARTE ${ }^{1}$ \\ Thaynara do Monte MÉLO ${ }^{1}$ \\ Hellen Cristina Barbosa PEREIRA ${ }^{1}$ \\ Yago Tavares PINHEIRO ${ }^{1}$ \\ Rielly Maria Cruz da SILVA2 \\ Jairo Domingos de MORAIS 3 \\ ${ }^{1}$ Fisioterapeutas pela Faculdade Mauricio de Nassau, UNINASSAU, 58030-000, João Pessoa - PB, Brasil
}

${ }^{2}$ Graduanda do curso de Fisioterapia da Faculdade Maurício de Nassau, UNINASSAU, 58030-000, João Pessoa - PB, Brasil ${ }^{3}$ Docente Mestre do Curso de Fisioterapia da Faculdade Maurício de Nassau, UNINASSAU, 58030-000, João Pessoa - PB, Brasil

\section{Resumo}

Introdução: A Intervenção Precoce apresenta-se como uma abordagem de caráter sistemático e sequencial que desenvolve e potencializa as atividades do cérebro da criança. Objetivo: Conhecer as variáveis clínicas e sociodemográficas de crianças com disfunções neurológicas atendidas no setor de intervenção precoce. Material e método: Trata-se de um estudo do tipo transversal, de caráter exploratório e com abordagem quantitativa, constituído por 42 cuidadores de crianças com idade entre 0 e 3 anos atendidas no setor de intervenção precoce da FUNAD (Fundação Centro Integrado de Apoio ao Portador de Deficiência), a coleta de dados foi realizada mediante aplicação de um questionário semiestruturado elaborado pelos pesquisadores. Resultados: Foi verificado que a maioria das famílias possuía renda mensal de até um salário mínimo. Notou-se que a maioria dos entrevistados possui ensino médio completo. A amostra foi composta, em maior número, por mães. Entre os pacientes predomina a faixa-etária de 12 a 17 meses, a microcefalia foi a patologia mais frequente. Conclusão: Maioria das crianças com alterações no desenvolvimento encontram-se em famílias com renda menor que um salário mínimo. É essencial o conhecimento do contexto familiar e social em que a criança está inserida.

Descritores: Desenvolvimento Infantil; Cuidadores; Perfil de Saúde.

\begin{abstract}
Introduction: Early Intervention presents itself as a systematic and sequential approach that develops and potentiates as activities of the child's brain. Objective: To know the clinical and sociodemographic variables of children with neurological dysfunction treated in the early intervention sector. Material and method: This is a cross-sectional exploratory study with a quantitative approach, consisting of 42 caregivers of children aged 0-3 in the early intervention sector of FUNAD (Fundação Deficiency Carrier), the data collection was performed through the application of a semistructured questionnaire prepared by the researchers. Results: It was verified that most families had a monthly income of up to one minimum wage. It was noted that the majority of respondents have completed high school. The sample was composed, in greater number, by mothers. Among patients, the age range was 12 to 17 months, microcephaly was the most common pathology. Conclusion: Most children with developmental changes are in families with incomes less than a minimum wage. Knowledge of the family and social context in which the child is inserted is essential.

Descriptors: Child Development; Caregivers; Health Profile.

\section{Resumen}

Introducción: La Intervención precoz se presenta como un abordaje de carácter sistemático y secuencial que desarrolla y potencian las actividades del cerebro del niño. Objetivo: Conocer las variables clínicas y sociodemográficas de niños con disfunciones neurológicas atendidas en el sector de intervención precoz. Material y método: Se trata de un estudio del tipo transversal, de carácter exploratorio y con abordaje cuantitativo, constituido por 42 cuidadores de niños con edad entre 0 y 3 años atendidos en el sector de intervención precoz de la FUNAD (Fundación Centro Integrado de Apoyo al Desarrollo), que se llevó a cabo mediante la aplicación de un cuestionario semiestructurado elaborado por los investigadores. Resultados: Se verificó que la mayoría de las familias tenían ingresos mensuales de hasta un salario mínimo. Se ha notado que la mayoría de los encuestados poseen una enseñanza media completa. La muestra fue compuesta, en mayor número, por madres. Entre los pacientes predomina la franja de edad de 12 a 17 meses, la microcefalia fue la patología más frecuente. Conclusión: Mayoría de los niños con alteraciones en el desarrollo se encuentran en familias con ingresos menores que un salario mínimo. Es esencial el conocimiento del contexto familiar y social en que el niño está inserto.

Descriptores: Desarrollo Infantil; Cuidadores; Perfil de Salud.
\end{abstract}

\section{INTRODUÇÃO}

O desenvolvimento infantil pode ser definido como um processo multidimensional e integral que se inicia com a concepção e inclui o desenvolvimento físico, a maturação neurológica, o desenvolvimento comportamental, motor, sensorial, cognitivo e de linguagem, assim como as relações socioafetivas. Tem como propósito tornar a criança capaz de responder às suas necessidades e as do meio tendo em vista o seu contexto de vida ${ }^{1}$.

Incontestavelmente, os primeiros anos de vida representam uma etapa primordial e determinante no desenvolvimento do ser humano. Nela se consolida toda a base para as aprendizagens posteriores, considerando que o crescimento e desenvolvimento, junto às experiências de interação com o ambiente permitirão a aprendizagem e o desenvolvimento de habilidades sociais, emocionais, cognitivas, sensoperceptivas e motoras, que serão a essência de toda uma vida ${ }^{2}$.

Pode-se afirmar que o desenvolvimento é global, contínuo, ordenado e depende do ritmo próprio de cada criança, ocorrendo no sentido cefalocaudal, próximo-distal, 
ventral-dorsal e global para o específico. Vale enfatizar que o desenvolvimento intelectual e afetivo são primordiais para a construção das habilidades motoras, que por sua vez, não podem ser analisadas isoladamente das funções sensoriais. As etapas do desenvolvimento motor evoluem gradativamente, sendo uma consequência da precedente e necessária para a aquisição da próxima etapa ${ }^{3}$.

Nesse seguimento, os fatores ambientais e biológicos, como a condição sócia econômica, podem influenciar o desenvolvimento de forma positiva ou negativa. $\mathrm{O}$ desenvolvimento da criança pode ser afetado de maneira negativa por vários fatores incidentes no período pré, peri e pós-natal, aumentando a probabilidade da criança apresentar alterações na aquisição de habilidades motoras, psicossociais e cognitivas ${ }^{4}$.

Quando há lesão no Sistema Nervoso Central (SNC), ocorre falha ou interrupção no desenvolvimento do sistema neuromaturacional resultando em ocorrências de padõres motores atípicos competindo com os padrões típicos, decorrente de um SNC que tenta se adaptar a uma situação inesperada. Caso os padrões motores atípicos prevaleçam o desenvolvimento global da criança é prejudicado. O atraso do desenvolvimento pode estar associado a várias condições da infância, resultante de fatores prejudiciais, como os agravos neurológicos e genéticos, ou uma condição transitória. Compreende-se, portanto que quanto mais rápida for à intervenção, menor será o impacto da morbimortalidade infantil, considerando que fatores ambientais podem sobrepor-se aos determinantes genéticos ${ }^{5-7}$.

Existem lesões potencialmente recuperáveis, desde que sejam traçados objetivos precisos de tratamento com níveis apropriados de estímulos facilitadores e inibidores. Partindo dessa premissa, a intervenção precoce surge como um programa de acompanhamento e intervenção clínicoterapêutica multiprofissional com bebês de alto risco e crianças na primeira infância acometidas por alguma patologia, buscando o melhor desenvolvimento possível por meio da mitigação de prováveis sequelas, além de promover efeitos benéficos na aquisição da linguagem e socialização, podendo contribuir na estruturação do vínculo mãe/bebê, na compreensão e no acolhimento familiar dessas crianças ${ }^{8,9}$.

Ressalta-se que os resultados de uma intervenção precoce são mais contundentes a partir do envolvimento e participação ativa da família. Para isso, é necessário levar em consideração valores e aspectos culturais de cada núcleo familiar além de promover uma aprendizagem colaborativa pautada no oferecimento de oportunidades à família ${ }^{8-13}$.

O convívio com crianças que apresentam alterações neurológicas mostrou-nos a necessidade de realizar estudos para melhor conhecer o perfil dessa população em nosso estado, haja vista a demanda de cuidados no âmbito hospitalar ou no cotidiano familiar. Essas crianças têm necessidade de cuidado profissional continuo e prolongado. O conhecimento do perfil das crianças com disfunções neurológicas oportuniza o aprofundamento do contexto familiar e social da criança, o que tem grande relevância na definição de estratégias para a reabilitação.

Desta maneira, o objetivo é conhecer as variáveis clínicas e sociodemográficas de crianças atendidas no setor de Intervenção Precoce.

\section{MATERIAL E MÉTODO}

Levando em consideração os objetivos do estudo em evidência e a forma como foi aplicado, o mesmo é considerado uma pesquisa de campo do tipo transversal, de caráter exploratório e com abordagem quantitativa, desenvolvido com crianças atendidas no programa de Intervenção Precoce da Fundação Centro Integrado de Apoio ao Portador de Deficiência (FUNAD), órgão estadual referência no Serviço de habilitação e reabilitação nas quatro áreas da deficiência - CER IV (física, intelectual, visual e auditiva) - onde os pacientes são atendidos por uma equipe multidisciplinar ofertada pela instituição.

A amostra selecionada por conveniência de forma não probabilística que envolveu a participação de 42 cuidadores de crianças atendidas com 0 a 3 anos de idade, de ambos os sexos e que apresentam disfunções neurológicas na FUNAD. Fora adotados como critérios de exclusão, cuidadores de crianças com mais de 3 anos e que recebiam tratamento há menos de 6 meses.

A coleta foi realizada no período de Março a Maio de 2017. Os dados foram colhidos utilizando-se questionário de caráter objetivo elaborado pelos pesquisadores.

O referido questionário (Quadro 1) compõe-se de quatro partes, englobando 13 questões e constando de uma parte introdutória com identificação do responsável/cuidador. A segunda parte contém questões que visam determinar o nível socioeconômico do público, subsequentemente à caracterização da criança e posteriormente informações quanto ao meio de transporte utilizado para chegar aos atendimentos.

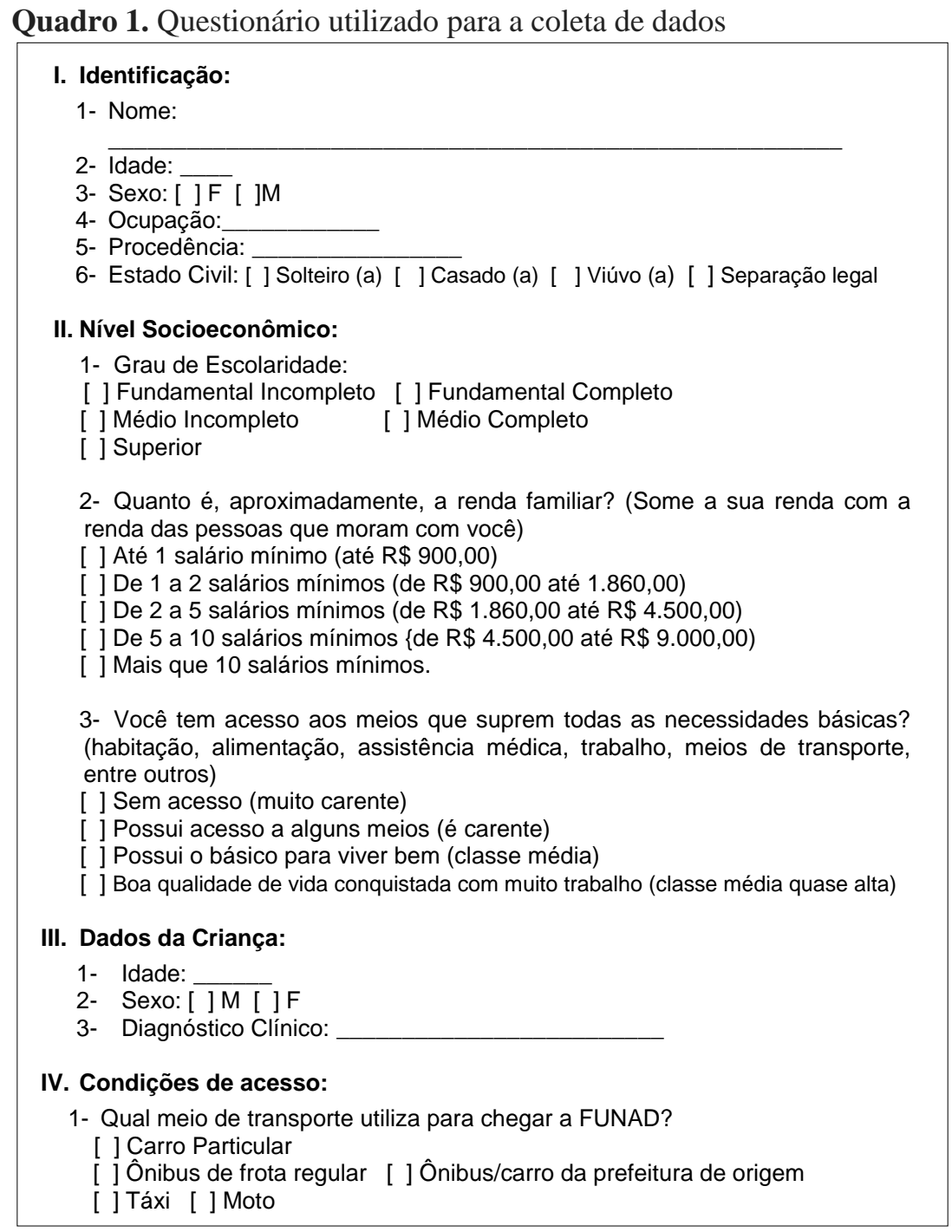

A análise de dados foi realizada através do programa SPSS 20.0 (Statistical Package for the Social Evidence) atribuindo nível de significância de 5\%.

O estudo foi desenvolvido dentro dos padrões éticos de pesquisa envolvendo seres humanos estabelecidos pela Resolução n 466/12 do Conselho Nacional de Saúde, tendo sido submetido e aprovado pelo Comitê de Ética, obtendo o parecer n ${ }^{\circ} 205 / 06613$.

A participação no referido estudo foi realizada de forma voluntária mediante a orientação sobre a pesquisa e assinatura do termo de consentimento livre e esclarecido TCLE pelos pais e/ou responsáveis participantes do estudo. 


\section{RESULTADOS}

De acordo com a Tabela 1, o estudo realizado demonstrou que $37(88,1 \%)$ dos cuidadores são do sexo feminino e $9(11,9 \%)$ do sexo masculino, com idade variando ente 19 anos e 60 anos. A análise do grau de parentesco do cuidador evidenciou a predominância significativa de mães $(83,3 \%)$. Em relação à ocupação observou-se que $34(81,0 \%)$ as cuidadoras são designadas como "do lar". Quanto à procedência notou-se que 23 $(54,8 \%)$ participantes são provenientes do interior da Paraíba e $18(42,9 \%)$ da capital do estado. A análise da situação conjugal constatou que $21(50 \%)$ dos cuidadores são solteiros e $19(45,2 \%)$ são casados.

Tabela 1. Caracterização dos cuidadores de crianças com disfunções neurológicas

\begin{tabular}{c|c|c|c}
\hline Variável & & $\mathbf{n}$ & $\mathbf{\%}$ \\
\hline \multirow{2}{*}{ Sexo } & Feminino & 37 & $88,1 \%$ \\
& Masculino & 5 & $11,9 \%$ \\
\hline \multirow{3}{*}{ Faixa Etária } & Até 24 anos & 8 & $19,0 \%$ \\
& De 24 a 35 anos & 22 & $52,4 \%$ \\
& Acima de 35 anos & 12 & $28,6 \%$ \\
\hline \multirow{5}{*}{ Ocupação } & Do lar & 34 & $81,0 \%$ \\
& Comerciante & 2 & $4,8 \%$ \\
& Estudante & 2 & $4,8 \%$ \\
& Aposentada & 1 & $2,4 \%$ \\
& Contador & 1 & $2,4 \%$ \\
& Professora & 1 & $2,4 \%$ \\
Procedência & Recepcionista & 1 & $2,4 \%$ \\
\hline \multirow{3}{*}{ Estado Civil } & Interior da Paraíba & 23 & $54,8 \%$ \\
& Capital da Paraíba & 18 & $42,9 \%$ \\
& Pernambuco & 1 & $2,4 \%$ \\
\hline \multirow{2}{*}{ Grau de parentesco } & Solteiro (a) & 21 & $50,0 \%$ \\
com a criança & Casado (a) & 19 & $45,2 \%$ \\
& Viúvo (a) & 1 & $2,4 \%$ \\
& Separação legal & 1 & $2,4 \%$ \\
\hline & Mãe & 35 & $83,3 \%$ \\
& Pai & 4 & $9,5 \%$ \\
& Avó & 2 & $4,8 \%$ \\
& Tia & 1 & $2,4 \%$ \\
\hline
\end{tabular}

Fonte: dados da pesquisa, 2017

A Tabela 2 resume os dados socioeconômicos dos cuidadores, onde maioria $(59,5 \%)$ relatou ter acesso a apenas alguns meios que suprem necessidades básicas (habitação, alimentação, assistência médica, trabalho e meios de transporte). Verificou-se que maioria dos entrevistados possuem ensino médio completo $(40,5 \%)$ e renda familiar de até 1 salário mínimo $(75,2 \%)$.

As informações adquiridas sobre o transporte utilizado para chegar aos atendimentos revelam que 21 $(50 \%)$ dos cuidadores utilizam ônibus ou carro da prefeitura de origem (Gráfico 1)

Tabela 2. Distribuição dos cuidadores segundo aspectos socieconômicos

\begin{tabular}{|c|c|c|c|}
\hline Variável & & $\mathbf{n}$ & $\%$ \\
\hline $\begin{array}{c}\text { Grau de } \\
\text { escolaridade }\end{array}$ & $\begin{array}{l}\text { Fundamental Incompleto } \\
\text { Fundamental Completo } \\
\text { Médio Incompleto } \\
\text { Médio Completo } \\
\text { Superior }\end{array}$ & $\begin{array}{c}14 \\
1 \\
7 \\
17 \\
3 \\
\end{array}$ & $\begin{array}{c}33,3 \% \\
2,4 \% \\
16,7 \% \\
40,5 \% \\
7,1 \% \\
\end{array}$ \\
\hline Renda familiar & $\begin{array}{c}\text { Até } 1 \text { salário mínimo } \\
\text { De } 1 \text { a } 2 \text { salários mínimos } \\
\text { De } 2 \text { a } 5 \text { salários mínimos }\end{array}$ & $\begin{array}{c}32 \\
8 \\
2\end{array}$ & $\begin{array}{c}75,2 \% \\
19,0 \% \\
4,8 \% \\
\end{array}$ \\
\hline $\begin{array}{l}\text { Acesso aos meios } \\
\text { que suprem } \\
\text { necessidades } \\
\text { básicas* }\end{array}$ & $\begin{array}{c}\text { Sem acesso (muito carente) } \\
\text { Acesso a alguns meios (é } \\
\text { carente) } \\
\text { Possui o básico para viver } \\
\text { bem (classe média) }\end{array}$ & $\begin{array}{c}1 \\
25 \\
16\end{array}$ & $\begin{array}{l}2,4 \% \\
59,5 \% \\
38,1 \%\end{array}$ \\
\hline $\begin{array}{l}\text { Meio de transporte } \\
\text { utilizado para chegar } \\
\text { aos atendimentos }\end{array}$ & $\begin{array}{c}\text { Ônibus/carro da prefeitura } \\
\text { de origem } \\
\text { Carro particular } \\
\text { Ônibus de frota regular } \\
\text { Táxi }\end{array}$ & $\begin{array}{l}21 \\
12 \\
7 \\
2\end{array}$ & $\begin{array}{c}50,0 \% \\
28,6 \% \\
16,7 \% \\
4,8 \%\end{array}$ \\
\hline
\end{tabular}

Fonte: dados da pesquisa, 2017
Gráfico 1. Distribuição dos cuidadores e crianças com disfunções neurológicas em relaçãp ao transporte utilizado para chegar ao atendimento

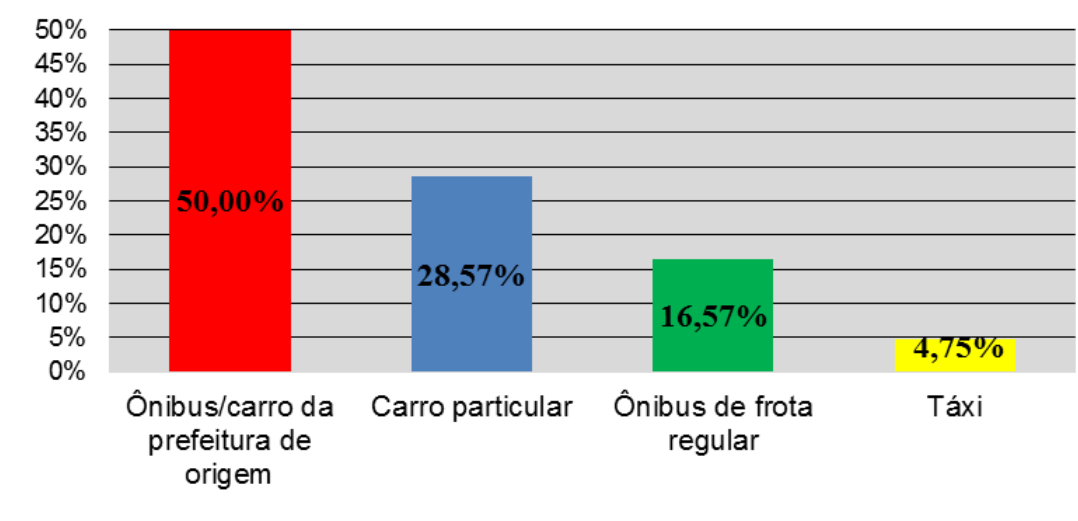

No que se refere à caracterização das crianças a Tabela 3 explana uma distribuição praticamente equivalente, com $22(52,4 \%)$ crianças do sexo masculino e $20(47,6 \%)$ do sexo feminino, a menor idade encontrada foi de nove meses e a maior idade foi 3 anos. Além disso, notou-se que $31,0 \%$ das crianças iniciaram a reabilitação com menos de três meses, porém, boa parte das crianças iniciaram os atendimentos após os 7 meses $(26,2 \%)$.

Tabela 3. Distribuição das crianças de 0 a 3 anos atendidas no setor de estimulação precoce conforme variáveis referentes à sexo, idade atual e idade que iniciou a reabilitação

\begin{tabular}{l|l|c|l}
\hline \multicolumn{1}{c|}{ Variável } & & $\mathbf{n}$ & \multicolumn{1}{c}{$\%$} \\
\hline Sexo & Masculino & 22 & $52,4 \%$ \\
& Feminino & 20 & $47,6 \%$ \\
\hline Idade (em meses) & De 9 a 11 meses & 2 & $4,8 \%$ \\
& De 12 a 16 meses & 19 & $45,2 \%$ \\
& De 17 a 23 meses & 17 & $40,5 \%$ \\
& De 24 a 36 meses & 4 & $8,5 \%$ \\
\hline Idade que iniciou a & $<3$ meses & 13 & $31,0 \%$ \\
reabilitação (em & 3 meses & 7 & $16,7 \%$ \\
meses) & $>3$ meses & 5 & $11,9 \%$ \\
& $>5$ meses & 6 & $14,3 \%$ \\
& $>7$ meses & 11 & $26,2 \%$ \\
\hline
\end{tabular}

Fonte: dados da pesquisa, 2017

De acordo com o diagnóstico clínico, obteve-se um total de 8 patologias (Tabela 4), entre elas, a microcefalia apresentou um elevado percentual, com $22(52,4 \%)$ dos casos entre os 42 pacientes. O segundo diagnóstico clínico com maior incidência foi a paralisia cerebral com $8(19,0 \%)$ pacientes. A síndrome de Down aparece como o terceiro diagnóstico clínico mais encontrado com 6 pacientes $(14,3 \%)$.

Tabela 4. Distribuição das crianças avaliadas em função do diagnóstico clínico

\begin{tabular}{c|c|c}
\hline Diagnóstico & $\mathbf{n}$ & $\mathbf{\%}$ \\
\hline Microcefalia & 22 & $52,4 \%$ \\
\hline Paralisia Cerebral & 8 & $19,0 \%$ \\
\hline Síndrome de Down & 6 & $14,3 \%$ \\
\hline Hidrocefalia & 2 & $4,8 \%$ \\
\hline Agenesia do corpo caloso & 1 & $2,4 \%$ \\
\hline Artrogripose & 1 & $2,4 \%$ \\
\hline Síndrome de Dandy Walker & 1 & $2,4 \%$ \\
\hline Síndrome de Moebius & 1 & $2,4 \%$
\end{tabular}

Fonte: dados da pesquisa, 2017

\section{DISCUSSÃO}

A predominância do gênero feminino demonstra que as próprias mães que se dedicam integralmente ao cuidado da criança, tendo abandonado suas atividades profissionais ou educacionais $\mathrm{O}$ que corrobora coma literatura, que destaca o papel da mulher como cuidadora ${ }^{14}$.

Entre os desafios enfrentados pelos cuidadores ressalta-se a estrutura financeira, acesso à rede de saúde 
para o atendimento da criança e a centralização da responsabilidade pelo cuidado sobre a mulher, mostrando uma condição de gênero associado ao cuidado à criança como uma tarefa exclusivamente feminina. Quando um dos pais, geralmente a mãe, necessita deixar o trabalho fora do lar para cuidar da criança, agrava a condição socioeconômica do grupo familiar ${ }^{15,16}$. A literatura relata que as condições econômicas são decisivas no desenvolvimento humano. É fundamental o conhecimento do contexto familiar e social em que a criança está inserida $^{17,18}$.

Os dados obtidos nos questionários mostram que $40,5 \%$ dos entrevistados apresentavam como grau de escolaridade nível médio completo, de acordo com os dados do IBGE 63,7 \% da população do estado da Paraíba possui menos de 8 anos de estudo ${ }^{19}$.

Segundo os dados do IBGE, a renda domiciliar per capita na Paraíba é menor que um salário mínimo. $\mathrm{O}$ resultado da presente pesquisa corrobora o de um estudo realizado por Aguiar et al. ${ }^{20}$ no qual a maioria das crianças com alterações no desenvolvimento encontravam-se em famílias com renda per capita menor que um salário mínimo ${ }^{19-20}$

Conforme os dados da Organização Mundial da Saúde (OMS), $10 \%$ da população de qualquer país é composta por algum tipo de deficiência, onde $4,5 \%$ corresponde à taxa entre aquelas com até 5 anos de idade que nascem ou adquirem impedimentos físicos, mentais ou sensoriais. No Brasil foi constatada a diminuição da prevalência de crianças com atraso do Desenvolvimento Neuropsicomotor (DNPM) em virtude do avanço nos cuidados neonatais e a expansão da assistência à criança nos primeiros anos de vida.

Esses mesmos fatores ocasionam uma situação paradoxal, uma vez que a maior sobrevivência de bebês de risco está relacionada ao aumento da morbidade, como as disfunções no neurodesenvolvimento. Dentre as disfunções neurológicas que mais acometem crianças de 0 a 3 anos podemos citar: encefalopatia crônica não progressiva da infância (paralisia cerebral), síndrome de Down e mielomeningocele ${ }^{21}$. No entanto, este estudo evidenciou uma alta predominância de crianças com diagnóstico de microcefalia. A microcefalia apresenta-se como uma malformação congênita onde o cérebro não se desenvolve de maneira adequada, caracterizada por um perímetro cefálico inferior ao esperado para a idade e sexo. Em 2015, o Brasil apresentou um surto de infecção pelo vírus Zika principalmente na Região Nordeste do Brasil, em todo território nacional foram consolidadas evidências que corroboram a presença do vírus Zika com o aumento da ocorrência da Microcefalia no país. O número de casos na Paraíba foi alarmante, visto a necessidade de maior atenção, dado como agravo de saúde pública ${ }^{8,22}$.

Sobre isso, vale enfatizar que além de medidas preventivas e atenção cuidadosa às gestantes, faz-se necessária à investigação do impacto emocional na qualidade de vida e rede de apoio às famílias das crianças incluídas como casos confirmados, bem como a capacitação de equipes multiprofissionais para avaliar e elaborar programas de intervenção ao longo do desenvolvimento da criança, principalmente nos três primeiros anos de vida. Os cuidadores parentais devem ser informados sobre a doença e seus desdobramentos, além disso, necessitam de abordagens educativas, grupos terapêuticos de apoio e treinamento para o cuidado diário ${ }^{23}$.
Dentre as crianças participantes do estudo, 26,2\% iniciaram os atendimentos no setor de estimulação precoce após os 9 meses. Apesar da capacidade de regeneração do organismo em qualquer idade, uma elevada plasticidade pode ser observada até 6 a 8 meses após o nascimento do bebê, fazendo com que este período seja fundamental para uma recuperação funcional mais próxima do normal ${ }^{24,25}$.

\section{CONCLUSÃO}

De acordo com os resultados obtidos por meio da análise do perfil das crianças com disfunções neurológicas, verificou-se que as condições socioeconômicas e o acesso à rede de saúde para realizar os atendimentos são as maiores dificuldades enfrentadas pelos familiares. As estratégias para a criação de ambientes favoráveis, objetivando a extensão da reabilitação em domicilio devem ser adaptadas às possibilidades específicas da família considerando seus aspectos socioeconômicos.

Portanto, é essencial conhecer o contexto em que a criança está inserida, bem como investigar o início do tratamento, sabendo-se que quanto mais rápida a intervenção melhor $\mathrm{o}$ prognóstico. $\mathrm{O}$ questionário foi considerado um instrumento útil na avaliação do perfil das crianças atendidas no programa de intervenção precoce.

$\mathrm{O}$ presente estudo trouxe importantes contribuições. Entretanto, novas pesquisas devem ser desenvolvidas em relação ao perfil clínico e sociodemográfico de crianças com alterações neurológicas.

\section{REFERÊNCIAS}

1. Organização Pan-Americana da Saúde. Manual para vigilância do desenvolvimento infantil no contexto da AIDPI. Washington, D.C; 2005.

2. OEA. Organização dos Estados Americanos. Primeira Infancia: Um olhar desde a neuroeducação, 2010. Disponível em: <http://iin.oea.org/pdf-iin/RH/primerainfancia-por.pdf $>$. Acesso em: 3 set, 2016.

3. Diament AJ, Cypel S. Neurologia Infantil. 4. ed. São Paulo: Atheneu; 2005.

4. Guardiola A, Egewarth C, Rotta NT. Avaliação do desenvolvimento neuropsicomotor em escolares de primeira série e sua relação com o estado nutricional. J Pediatr. 2001; 77(3):189-96.

5. Bax M, Goldstein M, Rosenbaum P, Leviton A, Paneth N, Dan B et al. Proposed definition and classification of cerebral palsy. Dev Med Child Neurol. 2005; 47(8):571-6.

6. Dornelas LF, Duarte NMC, Magalhães LC. Atraso do desenvolvimento neuropsicomotor: mapa conceitual, definições, usos e limitações do termo. Rev Paul Pediatr. 2015; 33(1):88-103.

7. Miranda LP, Resegue R, Figueiras ACM. A criança e o adolescente com problemas do desenvolvimento no ambulatório de pediatria. J Pediatr. 2003; 79(suppl1):S33-42.

8. Brasil. Ministério da Saúde. Secretaria de Atenção à Saúde. Diretrizes de Estimulação Precoce: Crianças de zero a 3 anos com Atraso no Desenvolvimento Neuropsicomotor Decorrente de Microcefalia. Brasília: Ministério da Saúde; 2016. 123p.

9. Reed UC. Paralisia cerebtal: neurologia. Ortopedia. Reabilitação. Arq Neuro-Psiquiatr. 2009; 67(2a):372-2.

10. Formiga CKMR, Pedrazzani ES, Tudella E. Desenvolvimento motor de lactentes pré-termo participantes de um programa de intervenção 
fisioterapêutica precoce. Rev Bras Fisioter. 2004; 8(3):239-45

11. Jingjing J, Li Q, Chu J, Zeng W, Yang M, Zhu S. Effect of $n-3$ PUFA supplementation on cognitive function throughout the life span from infancy to old age: a systematic review and metaanalysis of randomized controlled trials. Am J Clin Nutr. 2014; 6(3):1422-36.

12. Loureiro VR, Middleton S, Souza APD, Carbonel A, Vitorio A, Fuly LJPV et al. Intervenção precoce e estimulação global de crianças com deficiências e atrasos no desenvolvimento neuropsicomotor: um programa de extensão. RAI RUM. 2015; 3(1):187-204.

13. Pereira VA, Chiodelli T, Rodrigues OMPR, Silva CSO, Mendes VF. Desenvolvimento do bebê nos dois primeiros meses de vida: variáveis maternas e sociodemográficas. Pensando fam. 2004; 18(1):64-77.

14. Lackey NR, Gates MF. Adults' recollections of their experiences as young caregivers of family members with chronic physical illnesses. J Adv Nurs. 2001; 34(3): 320-8.

15. Lira BNR. Brava gente da Mangueira: a enfermagem desvendando o discurso de jovens sobre o cuidar do bebê prematuro na adolescência [dissertação]. Rio de Janeiro: Universidade Federal do Rio de Janeiro; 2005.

16. O'Brien ME. Living in a house of cards: family experiences with long-term childhood technology dependence. J. Pediatr Nurs. 2001; 16(1):13-22.

17. Souza SC. Avaliação do desenvolvimento neuropsicomotor do pré-escolar de creches públicas de Cuiabá-MT [dissertação]. São Paulo: Faculdade de Medicina da Universidade de São Paulo; 2003.

18. Sucupira ACSL, Werner JJr, Resegue R. Desenvolvimento. Sucupira ACSL, Bricks LF, Kobinger MEBA, Saito MI, Zuccoltto SMC (eds). Pediatria em consultório. 4. ed. São Paulo: Sarvier; 2000. p. 22-39.

19. IBGE. Instituto Brasileiro de Geografia e Estatística. Censo de 2016.

20. Aguiar LCV, Reis LL, Furieri N, Nascimento RC, Neves Jr JAS, Salles FL et al. Prevalência de atrasos no desenvolvimento neuropsicomotor em crianças de 06 a 24 meses. In: XI Encontro Latino Americano de Iniciação Científica e VII Encontro Latino Americano de Pós-Graduação - Universidade do Vale do Paraíba. 2007 Out 18-19; São José dos Campos, Brasil. p.1588-92.

21. Moura DR, Costa JC, Santos IS, Barros AJ, Matijasevich A, Halpern R et al. Natural history of suspected developmental delay between 12 and 24 months of age in the 2004 Pelotas birth cohort. J Paediatr Child Health. 2010; 46(6):329-36

22. Tavares EC, Barbosa AM, Portp MO, Silva VM, Holanda IMB.Visão epidemiológica da microcefalia em João Pessoa, Paraíba. In: Congresso Brasileiro de Ciências da Saúde. 2016; Campina Grande, Brasil.

23. Brunoni D, Blascovi-Assis SM, Osório AA, Seabra AG, Amato CA, Teixeira MC et al. Microcephaly and other Zika virus related events: the impact on children, families and health teams. Cienc Saude Colet. 2016; 21(10): 3297-302.

24. Basu AP. Early intervention after perinatal stroke: Opportunities and challenges. Dev Med Child Neurol. 2014; 56(6):516-21.

25. Yang JF, Livingstone D, Brunton K, Kim D, Lopetinsky B, Roy F et al. Training to enhance walking in children with cerebral palsy: are we missing the window of opportunity? Semin Pediatr Neurol. 2013; 20(2):106-15.

\section{CONFLITO DE INTERESSES}

Os autores declaram não haver conflitos de interesse.

\section{AUTOR PARA CORRESPONDÊNCIA}

\section{Natália de Fátima Pereira Meireles}

nataliameireles94@gmail.com

Submetido em 08/09/2017

Aceito em 04/10/2017 